\title{
Transjecting growth hormone: continuous nightmare or controlled nuisance? Evaluation of a new needle-free device
}

This article was published in the following Dove Press journal:

Patient Preference and Adherence

23 July 2013

Number of times this article has been viewed

\section{Ad A Kaptein}

Medical Psychology Section, Leiden University Medical Centre, Leiden, Netherlands
Correspondence: Ad A Kaptein

Medical Psychology Section, Leiden

University Medical Centre (LUMC),

PO Box 9600, Leiden 2300 RC,

Netherlands

Tel + 3I 7I 5262905

Fax $+3|7| 5248123$

Email a.a.kaptein@lumc.nl
Background: Administering growth-hormone therapy (GHT) is a long-term treatment, associated with avoidance and phobic behaviors in the children involved. The current study examined GHT users' perceptions of a new needle-free device (ZomaJet Vision X [10 mg/mL]) with a lower injection volume compared to the traditional device.

Methods: A total of 73 persons participated (mean age \pm standard deviation, $10.10 \pm 3.60$ years) in a longitudinal design. Users' views were studied 4 weeks after having applied both the old and the new device for a period of at least 4 weeks. Satisfaction, ease and frequency of restitution, local sensations, bruises during administering GHT, affective response to local sensations, and subject preference were assessed on the basis of the users' responses.

Results: Subjects' satisfaction with the new device was equal compared with the previous device for the total group of 73 children. However, the subgroup of 59 children who proved tolerant to meta-cresol (new preservative for Vision $\mathrm{X}$ only) reported a significantly higher satisfaction rating with the new device compared to the old device ( 7.7 vs $6.6, P=0.0002$ ). Vision $\mathrm{X}$ was evaluated as better on ease and frequency of restitution and the number of bruises. Pain sensations did not differ meaningfully between the two devices. The new device was favored over the previous one in a majority of respondents. Vision $\mathrm{X}$ allows easy reconstitution of the solution, which was reflected in the percentage of young children able to prepare transjections themselves being more than doubled, illustrating the greater sense of empowerment in these users. Self-reported adherence to the therapy was good (less than $10 \%$ of injections missed) with both devices.

Conclusion: The new device ZomaJet Vision X appears to be evaluated more positively than the previous version on criteria that reflect users' preferences.

Keywords: invasive medical procedures, growth-hormone therapy, patient perception, transjection, children

\section{Introduction}

One needs only to study Renaissance paintings and etchings depicting invasive or therapeutic medical procedures to witness the horror and pain of the patients undergoing such procedures. In more modern times, physicians, behavioral scientists, and producers of medical equipment are doing their utmost to minimize discomfort, pain, and fear, potentially associated with undergoing invasive diagnostic or therapeutic medical procedures.

Needles in a medical application elicit fear, disgust, and anticipatory pain in a large proportion of persons. ${ }^{1}$ Having to undergo a venipuncture, getting an intravenous needle inserted in a vein in the arm, or being subjected to a set of injections in the context of a vaccination for a trip to the tropics, is hardly a nonevent for anyone. 
Therapeutic approaches have been developed to a great level of sophistication, resulting in lower levels of fear, pain, and avoidance behavior. $^{2}$

Long-term use of growth-hormone therapy (GHT) is used for treatment of children with growth disturbances due to various etiologies, such as chronic renal failure, growth-hormone deficiency, born small for gestational age, or Turner's syndrome. GHT appears to be associated with gains in body length of on average $8-11 \mathrm{~cm}$ in children with growth-hormone deficiency. ${ }^{3}$ From a behavioral medicine point of view, a biomedical conceptualization of GHT seems a simplification of reality: adherence to daily injection of GHT is far from optimal, fueled by not wanting to be confronted daily with a medical problem, by fear of injections, and because of balancing daily medical actions vs an increase in body length in a future that is perceived as far off. ${ }^{4}$ Persons in whom GHT is prescribed are required to inject themselves or to have a parent or caregiver deliver the injections daily for an extended length of time. These conditions are hardly conducive to achieving optimal "compliance" with actually applying the prescribed injections.

A recent study on views of parents on GHT for their children corroborated these findings. Almost half of the children showed anxiety about the needle or the pain associated with administering the injection; parents reported welcoming support about how to deal with this anxiety and adherence issues. ${ }^{5}$ In addition to behavioral medicine interventions focusing on pain-reduction techniques ${ }^{6}$ and patient-empowerment approaches, ${ }^{7}$ attempts to design injection systems aimed at minimizing pain and discomfort are a third method. Wickramasuriya et al examined factors associated with patients' preferences regarding GHT devices. ${ }^{8}$ It was found that ease of use was the major determinant of preference. Also, boys preferred light-blue devices. In this context, we examined the evaluation of patients and their caregivers of a new injection device. This device (ZomaJet Vision X [10 mg/mL]; Ferring Pharmaceuticals, Saint-Prex, Switzerland) transjects growth hormones through the skin of the user, ie, no needles are involved. In this paper, we describe patients' preferences and views regarding two ZomaJet devices, ie, the ZomaJet 2 Vision vs the ZomaJet Vision X. Both devices are based on the same jet-injection technique. However, the new device allows injection of more concentrated growth hormone, resulting in a threefold-lower injection volume compared to the previous device. Moreover, the new device comes with a prefilled syringe, as a result of which it is no longer necessary to break ampoules during the reconstitution of growth hormone.

\section{Materials and methods}

\section{Study population}

Study subjects were children and adolescents who were prescribed GHT due to delayed growth as a consequence of severe growth-hormone deficiency, Turner's syndrome, chronic renal failure, or who were born small for gestational age. Subjects were selected by physicians from ten outpatient departments of pediatrics in The Netherlands.

The study was designed as a noninterventional, multicenter survey in a nonrandomized sample. Subjects had been prescribed standard GHT and standard care for a minimum of 36 months (no changes in treatments were required for this survey). They were offered the use of a new needle-free device - the ZomaJet Vision X (Figure 1, right). The use of the ZomaJet Vision X was voluntary.

The intention-to-treat (ITT) population consisted of all subjects included in the survey from whom written informed consent had been obtained $(n=126)$. The per-protocol (PP) population consisted of all subjects fully completing the primary end point of the survey. A total of 73 subjects were included in this population. These subjects switched from ZomaJet 2 Vision to ZomaJet Vision X and completed both visits of the survey. The total number of subjects who completed both visits was lower than expected. This was probably due to the long period between subject inclusion and the launch of the Zomajet Vision X (up to 2 years).

In the PP population, 14 subjects experienced a burning sensation at the injection site after injection using the ZomaJet Vision X. This could be an indication that they were intolerant to meta-cresol (m-cresol). $\mathrm{m}$-Cresol is the preservative in the Zomacton solution that is used in combination with the Vision $\mathrm{X}$ device. The experience of these subjects

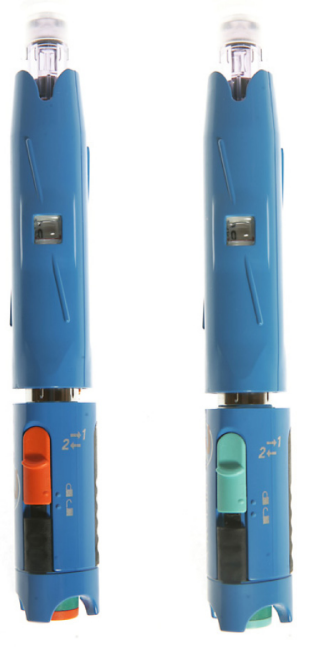

Figure I Zomajet 2 Vision (left) and Zomajet Vision X (right). 
was based predominantly on the adverse effect of the applied medicine, which obscured the analysis of the perception of the device (which was the main study objective). Therefore, an additional population was defined and analyzed, excluding these 14 subjects from the PP population. This population was called the nonswitched population (NSP). The ITT population consisted of 126 subjects, and the PP and NSP groups 73 and 59, patients respectively.

\section{Assessments}

Patients on the old device completed questionnaire 1 and diary 1 (14-day period) at visit 1 (directly after informed consent). When the ZomaJet Vision X became available, subjects could choose to switch to this new pen (= visit 2 ). Four weeks after they started using the new device, they were asked to complete questionnaire 2 and diary 2 (14-day period).

The primary end point - overall subject satisfaction - was assessed in questionnaire 1 at visit 1 for ZomaJet 2 Vision, and in questionnaire 2 (after visit 2 ) for both pens (ZomaJet 2 Vision retrospectively). Subjects had to circle a number between 1 and 10, where 1 indicated not satisfied at all and 10 indicated very satisfied.

For the assessment of the secondary end points, the subjects answered several identical questions in the questionnaire and diary 1 and 2 that addressed their views and perceptions of the subjects on:

- ease and frequency of reconstitution (= preparing the transjection)

- painful sensations and bruises during administering growth hormone

- affective response to painful sensation and bruises

- overall preference.
Differences between scores of the respondents in the two device categories were tested with Student's $t$-test.

\section{Results}

The results pertain to 73 children, with a mean age (standard deviation) of 10 (3.6) years. Primary school and vocational school were attended by about $70 \%$ of the subjects; the others attended higher education or some other type of education. There were no differences in mean age and educational level between the three analysis samples (ITT, PP, and NSP). The mean satisfaction ratings at visit 2 are depicted in Figure 2. In the NSP, subjects reported an increase in satisfaction with the ZomaJet Vision X.

The new device, ZomaJet Vision X, was evaluated more positively (higher overall satisfaction) than the previous device, ZomaJet Vision 2: 6.8 vs $7.7(P<0.0002)$ in the NSP. In the PP sample, no meaningful differences were discerned (mean scores 6.8 vs 7.1).

These results are in accordance with the results of asking the children to compare the handling with both devices and the response to the question on the age that children did all the handling themselves, independent from caregivers.

When comparing the difficulty of the preparation of the solution, the handling that came with the new device was perceived (much) better by $87.5 \%$ of the users (ITT population), while only $7 \%$ were of the opposite opinion. This preference for the new device was also reflected in the responses to the question: "How difficult was the preparation of the solution?" (Figure 3).

An important aspect of encouraging empowerment in the children who are using GHT pertains to the age at which they prepare the solution themselves. Children younger than

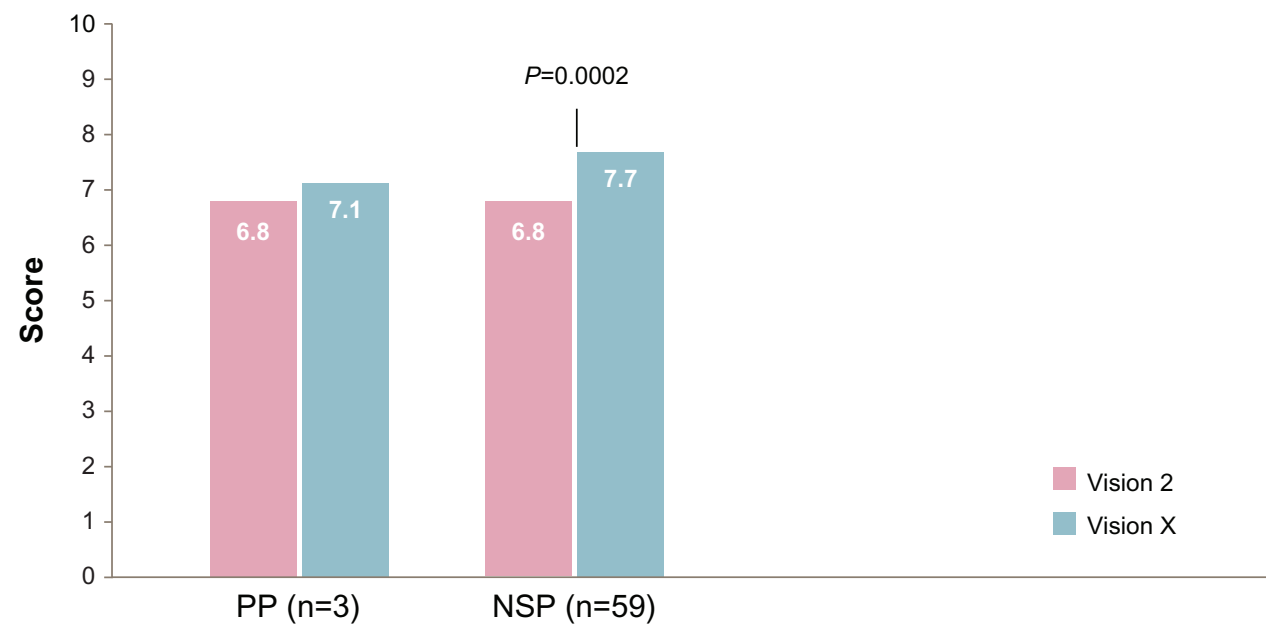

Figure 2 Mean satisfaction score in the two samples.

Abbreviations: PP, per protocol population; NSP, nonswitched population. 


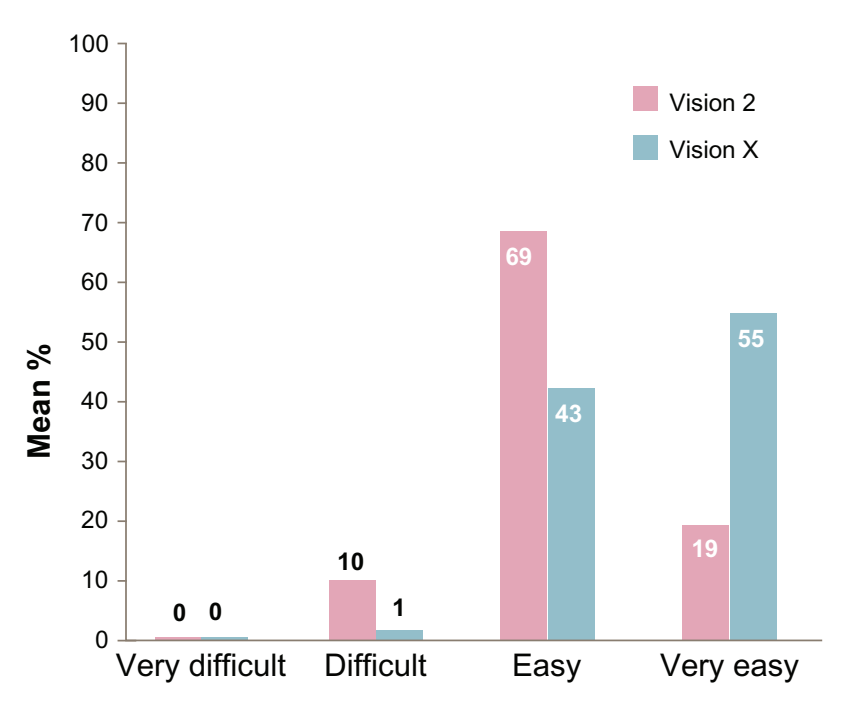

Figure 3 Grade of difficulty preparing the solution in the per protocol population (PP).

10 years hardly prepared the solution themselves. Between the ages of 10 and 12 years, $32 \%$ of the Vision X users (PP) reported preparing the solution themselves, with only 5.3\% of Vision 2 users doing this. In children older than 12 years, these figures were $47 \%$ and $18 \%$, respectively.

During the injection, users experienced pain to some extent. Our respondents indicated the degree of pain (none, a little, much) over a 14-day period, comparing the two transjection systems. As can be seen in Figure 4, the majority in both transjection systems reported experiencing no pain. Vision $\mathrm{X}$ appears to be associated with a somewhat higher degree of pain than the Vision 2 system.

Bruises turned out to be an infrequent consequence of the transjection systems. Respondents reported lower frequency of bruises over a 14 day period when using Vision X. Of the PP, $80 \%$ (Vision X) and 68\% (Vision 2) answered no to the question of whether or not they experienced some kind of bruising after the injection of the day before.
When asked about whether the bruises - if any - bothered them, $6.5 \%$ of all users indicated that the bruises did indeed bother them. The vast majority, however, (93.5\%) were not bothered about bruises that they had experienced. Bruising therefore seems of minor importance when evaluating the properties and the preference for these transjection systems. It should be noted that in both device systems there is no need to hold the device against the skin after the transjection.

\section{Overall preference}

Table 1 shows the overall preference in both subsamples. In both PP and NSP, the new device, Vision X, was perceived as preferable over the existing device, the ZomaJet 2.

The majority of the patients $(74.6 \%, n=50$, PP sample) preferred the new device over the older version. In patients that experienced no adverse effects caused by the m-cresol in the injection solution (the NSP sample), the difference was even more pronounced: $87.3 \%(\mathrm{n}=48)$ preferred the Vision X device.

\section{Discussion}

Users of a new device for the administration of GHT reported higher satisfaction overall with the device over an older version. These differences were observed in the subgroup of users who proved tolerant to $\mathrm{m}$-cresol. The patients felt more satisfied with the new device, felt that ease of use was much greater, reported fewer bruises, and clearly preferred the newer device over the somewhat older version. Of particular interest was the finding that at the age of $10-12$ years, $32 \%$ of children were able to prepare the medicine without help from their parents. In the age-group $12+$, this was $47 \%$, compared to $5 \%$ and $18 \%$, respectively, in the same age-groups when using the older version. This indicates improved empowerment of patients when using the new device.

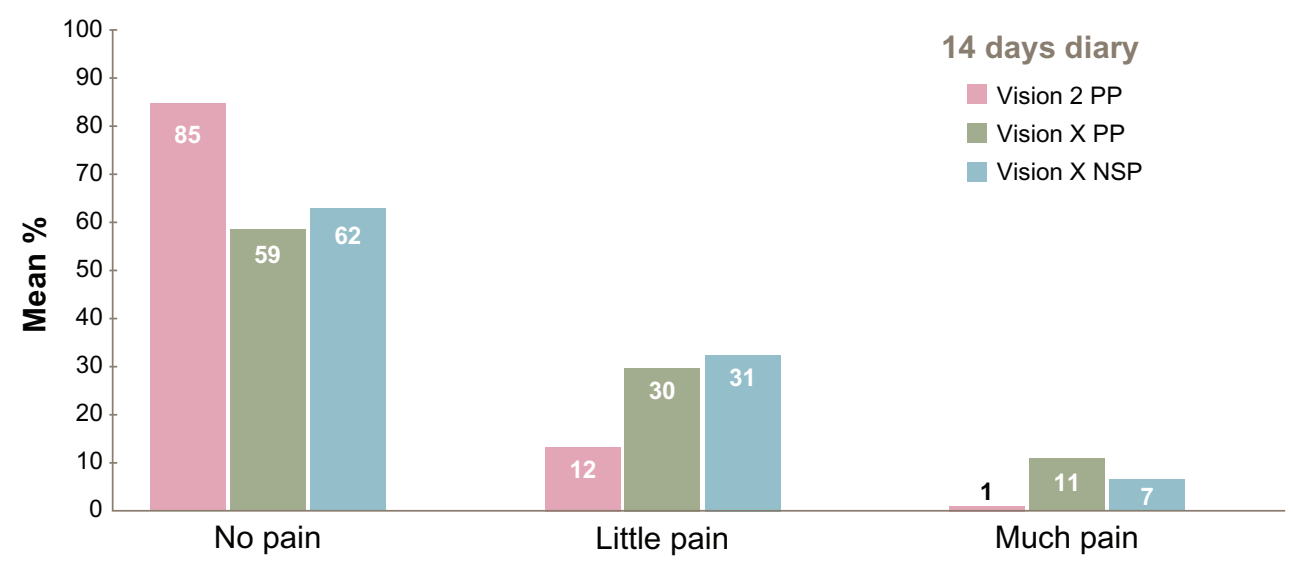

Figure 4 Degree of pain experienced during the transjection.

Abbreviations: PP, per protocol population; NSP, nonswitched population. 
Table I Overall preference for applying growth-hormone treatment

\begin{tabular}{lll}
\hline & PP $(n=73)$ & NSP $(n=59)$ \\
\hline $\begin{array}{l}\text { Which pen do you prefer to use for your hormone teatment } \\
\text { New vision X } \\
\mathrm{n}(\%)\end{array}$ \\
$\begin{array}{l}\text { Old vision } \\
\mathrm{n}(\%)\end{array}$ \\
$\begin{array}{l}\text { Missing } \\
\mathrm{n}\end{array}$ & $50(75)$ & $48(87)$ \\
\hline
\end{tabular}

Abbreviations: PP, per protocol population; NSP, nonswitched population.

In earlier work, Verrips et $\mathrm{al}^{9}$ found that a needle-free injector system was preferred by children on GHT. Research on the perception of invasive diagnostic and therapeutic procedures seems to emphasize the importance of the user/patient having or getting control over the procedures (eg, for a new pump system in patients with type 1 diabetes ${ }^{10}$ [see also Bailey and Campos, ${ }^{11}$ Blair et al, ${ }^{12}$ and Money et $\mathrm{al}^{13}$ for people who must autoinject epinephrine ${ }^{14}$; on migraine patients delivering subcutaneous sumatriptan ${ }^{15}$; about perceptions of pen injectors for diabetes management in type 2 diabetes patients ${ }^{16}$; on disposable autoinjector devices in patients with chronic hepatitis ${ }^{17,18}$; and about autoinjection devices for people with multiple sclerosis). In dentistry, almost anyone knows how lifting a hand indicates to the dentist that the patient wants the procedure to stop immediately. Dentist and patients seem perfectly happy with this empowering of patients. Our results tend to be in line with this finding: giving users and/or their caregivers control over administering the growth-hormone injection seems to be appreciated by user and caregiver, ${ }^{19}$ which has a positive impact on patient adherence. ${ }^{20}$

Our paper has a number of limitations. The design of the study had an observational character; a more experimental design can be designed based on our current study. In further research, patients on GHT could be randomized to a group that continued using the old device and a group that would be using the new device, preferably using medication with an identical preservative. This would allow better comparison of the two devices. Additionally, the assessment of the degree of satisfaction with a transjection system might benefit from a more detailed and specific operationalization of the concept of satisfaction. Research shows how global assessment of satisfaction tends to lead to a higher satisfaction score, compared to asking about satisfaction about specific components of medical care or a medical device. ${ }^{21}$ An additional issue that needs to be addressed in future research on this subject relates to a more detailed characterization of the respondents in the non-PP condition (diagnostic categories). In the study by Wickramasuriya et al, ${ }^{8}$ the color of the device turned out to be a determinant of patient preference. The new device in our study had a bluish hue; this may have caused boys' increased appreciation of this device. A strength of our study relates to the outcome measures used: they were virtually all patient-reported outcomes and patient-important outcomes. It goes without saying that a more biomedical outcome, eg, body length after a considerable period of administering GHT, would almost be the ultimate outcome measure of patient preference. However, this raises other issues, such as adherence to long-term use of growth hormone, irrespective of type of device.

Future research about patients' views of diagnostic and therapeutic invasive procedures would benefit quality of life of patients, caregivers, and health-care professionals. Incorporating these future findings in clinical guidelines seems an important, feasible, and humane objective. ${ }^{22}$

\section{Disclosure}

Ferring BV Hoofddorp, Netherlands, provided the organizational and financial means for the conduct of the study and the writing of the manuscript. The author reports no other conflicts of interest in this work.

\section{References}

1. Andrews GJ. 'I had to go to the hospital and it was freaking me out' needle phobic encounter space. Health Place. 2011;17:875-884.

2. Duff AJ, Gaskell SL, Jacobs K, Houghton JM. Management of distressing procedures in children and young people: time to adhere to the guidelines. Arch Dis Child. 2012;97:1-4.

3. National Institute for Health and Care Excellence. Human growth hormone (somatropin) for the treatment of growth failure in children (review) (TA188). 2010. Available from: http://guidance.nice.org.uk/ TA188. Accessed June 5, 2013.

4. Rosenfeld RG, Bakker B. Compliance and persistence in pediatric and adult patients receiving growth hormone therapy. Endocr Pract. 2008; 14:143-154.

5. van Dongen N, Kaptein AA. Parents' views on growth hormone treatment for their children: psychosocial issues. Patient Prefer Adherence. 2012;6:547-553.

6. Elverdam B. 'It is only a pinprick': (or is it?): childhood vaccinations in general practice as 'matter out of place.' Anthropol Med. 2011;18: 339-350.

7. Chewning B, Bylund CL, Shah B, Arora NK, Gueguen JA, Makoul G. Patient preferences for shared decisions: a systematic review. Patient Educ Couns. 2012;86:9-18.

8. Wickramasuriya BPN, Casey A, Akhtar A, et al. Factors determining patient choice of device for GH therapy. Horm Res. 2006;65:18-22.

9. Verrips GH, Hirasing RA, Fekkes M, Vogels T, Verloove-Vanhorick SP, Delamarre-van de Waal HA. Psychological responses to the needle-free Medi-Jector or the multidose Disetronic injection pen in human growth hormone therapy. Acta Paediatr. 1998;87:154-158.

10. Lebenthal Y, Lazar L, Benzaquen H, Shalitin S, Phillip M. Patient perceptions of using the Omnipod system compared with conventional insulin pumps in young adults with type 1 diabetes. Diabetes Technol Ther. 2012;14:1-7. 
11. Bailey T, Campos C. FlexTouch for the delivery of insulin: technical attributes and perception among patients and healthcare professionals. Expert Rev Med Devices. 2012;9:209-217.

12. Blair JC, Peak M, Gregory JW. What is the best way to deliver subcutaneous insulin to infants, children, and young people with type 1 diabetes mellitus? BMJ. 2011;343:d5221.

13. Money AG, Barnett J, Kuljis J, Lucas J. Patient perceptions of epinephrine auto-injectors: exploring barriers to use. Scand J Caring Sci. 2013;27(2):335-344.

14. Moore JC, Miner JR. Subcutaneous delivery of sumatriptan in the treatment of migraine and primary headache. Patient Prefer Adherence. $2012 ; 6: 27-37$

15. Toscano D, Brice J, Alfaro C. Usage and perceptions of pen injectors for diabetes management: a survey of type 2 diabetes patients in the United States. J Diabetes Sci Technol. 2012;6:686-694.

16. Varunok P, Lawitz E, Beavers KL. Evaluation of pharmacokinetics, user handling, and tolerability of peginterferon alfa-2a (40 kDa) delivered via a disposable autoinjector device. Patient Prefer Adherence. 2011;5: $587-599$.
17. Verdun E, Russell S, Snow T. Understanding and meeting injection device needs in multiple sclerosis: a survey of patient attitudes and practices. Patient Prefer Adherence. 2011;5:173-180.

18. d'Arcy C, Thomas D, Stoneman D, Parkes L. Patient assessment of an electronic device for subcutaneous self-injection of interferon $\beta$-1a for multiple sclerosis: an observational study in the UK and Ireland. Patient Prefer Adherence. 2012;6:55-61.

19. Gaskell S. Evidence-Based Guidelines for the Management of Invasive and/or Distressing Procedures with Children. Leicester: British Psychological Society; 2009.

20. Sabate E. Adherence to Long-Term Therapy: Evidence for Action. Geneva: WHO; 2003.

21. Eveleigh RM, Muskens E, van Ravesteijn H, van Dijk I, van Rijswijk E, Lucassen P. An overview of 19 instruments assessing the doctor-patient relationship: different models or concepts are used. J Clin Epidemiol. 2012;65:10-15.

22. Haverkamp F, Gasteyger C. A review of biopsychosocial strategies to prevent and overcome early-recognized poor adherence in growth hormone therapy of children. J Med Econ. 2011;14:448-457.
Patient Preference and Adherence

\section{Publish your work in this journal}

Patient Preference and Adherence is an international, peer-reviewed, open access journal focusing on the growing importance of patient preference and adherence throughout the therapeutic continuum. Patient satisfaction, acceptability, quality of life, compliance, persistence and their role in developing new therapeutic modalities and compounds to

\section{Dovepress}

optimize clinical outcomes for existing disease states are major areas of interest. This journal has been accepted for indexing on PubMed Central. The manuscript management system is completely online and includes a very quick and fair peer-review system. Visit http://www.dovepress.com/ testimonials.php to read real quotes from published authors. 